\title{
Improvement of the chilling tolerance of 'Monika' cucumber seedlings by short-term temperature and seed conditioning with plant growth regulators
}

\author{
Krzysztof Górnik \\ Department of Ornamental Nursery and Seed Science \\ Research Institute of Horticulture \\ Konstytucji 3 Maja 1/3, 96-100 Skierniewice, Poland
}

\begin{abstract}
The aim of the present study was to improve the chilling tolerance of cucumber seedlings. The conditioned seeds in solutions of salicylic or jasmonic acid in concentrations of $10^{-2}, 10^{-3}$ or $10^{-4} \mathrm{M}$ or brassinolide in concentrations of $10^{-6}, 10^{-8}$ or $10^{-10} \mathrm{M}$ were subjected to temperature shock of $0,2.5,5,35,40$ or $45^{\circ} \mathrm{C}$ for 1 , 2 or 4 hours. Seedlings with $3 \mathrm{~mm}$ roots were chilled at $0^{\circ} \mathrm{C}$ for three days. The chilling susceptibility was evaluated by measurements of roots lengths, electrolyte leakage and total dehydrogenase activity.

The obtained results indicated that cucumber tolerance to chilling conditions depended on the plant growth regulators used during seed imbibition and its concentration as well as the temperature initiating thermal shock and its duration. The highest tolerance to chilling, expressed by the subsequent growth of roots, was observed after seed conditioning in salicylic acid solutions in a concentration of $10^{-4} \mathrm{M}$ for $16 \mathrm{~h}$ at $25^{\circ} \mathrm{C}$, then exposed for $4 \mathrm{~h}$ to a shock temperature of $5^{\circ} \mathrm{C}$. The length of seedling roots after such treatment was over 12 times longer than the control (imbibed in distilled water and not subjected to short-term temperature impact). An increased chilling tolerance of cucumber seedlings may result from enhanced membrane integrity and total dehydrogenase activity. Further research is needed to explain the mechanism of the positive effects of salicylic acid, jasmonic acid or brassinolide application to reduce the injurious effects of chilling on cucumber seedlings.
\end{abstract}

Key words: brassinolide, Cucumis sativus L., electrolyte leakage, jasmonic acid, salicylic acid, temperature stress, total dehydrogenase activity

\section{INTRODUCTION}

Cucumber (Cucumis sativus L.), due to its origin, belongs to the group of plants with high thermal requirements (Saltveit 1991). In Poland, cucumbers grown in the field are exposed to adverse environmental factors, such as spring frosts and prolonged cold weather. This results in the inhibition of plant growth, yellowing of leaves and an increase in the occurrence of diseases, which in turn leads to the inferior quality of the crop or the death of plants.

Chilling temperature during seed germination and seedling emergence results in a reduction in seed germination and vigor, the slow growth of seedlings and their dehydration, necrosis and discoloration, greater sensitivity of plants to disease, inhibition of their growth, damages the 
root tips and ultimately their decay (Stark et al. 1993, Kacperska 2002). The adverse chilling effect observed at the cellular level concerned a loss of cytoplasm liquidity, a decrease in membrane integrity, changes in enzyme activity, accumulation of harmful compounds, increased release of carbon dioxide and ethylene and the reduction of photosynthesis. These symptoms increase with a decrease in temperature and the lengthening of the time of its impact (Saltveit 1991, Collins at al.1993, Jennings and Saltveit 1994, Mangrich and Saltveit 2000, Kang and Saltveit 2001, Saltveit 2001, Kang and Saltveit 2002).

Some attempts have been taken to increase seedling tolerance to chilling conditions. These methods include: temperature conditioning, storage at high relative humidity or low pressure, application of specific chemical compounds, use of controlled atmospheres during chilling, increased atmospheric carbon dioxide levels, pre-treatments with calcium or ethylene and conditioning near the chilling temperature before storage (Collins et al. 1993, Mangrich and Saltveit 2000, Kang and Saltveit 2001, Saltveit 2001). However, these methods were mainly used during the early growth of plants, which creates difficulties in applying them in practice during seed production.

Therefore, the aim of the present study was to evaluate the effect of temperature shock applied during seed conditioning in water or in the presence of salicylic acid, jasmonic acid or brassinolide on the improvement of cucumber tolerance to the negative effects of chilling.

\section{MATERIAL AND METHODS}

Seeds of cucumber (Cucumis sativus L.) were purchased from TORSEED S.A. - Garden Seed and Nursery Stock Company in Torun. Cucumber seed lots were kept at $30 \% \mathrm{RH}$ at $25^{\circ} \mathrm{C}$ until the start of the experiments.

The seeds were sown in $21.5 \times 15 \mathrm{~cm}$ Plexiglas boxes on cotton wool moistened with either distilled water at $25^{\circ} \mathrm{C}$ (control) or solutions of salicylic or jasmonic acid in concentrations of $10^{-2}, 10^{-3}$ or $10^{-4}$ $\mathrm{M}$ or brassinolide in concentrations of $10^{-6}, 10^{-8}$ or $10^{-10} \mathrm{M}$. Imbibed seeds were transferred to $0,2.5,5$, 35,40 or $45^{\circ} \mathrm{C}$ for $0-$ control, 1,2 or $4 \mathrm{~h}$ and returned to $25^{\circ} \mathrm{C}$. Seedlings with $3 \mathrm{~mm}$ roots were chilled at $0^{\circ} \mathrm{C}$ for three days. The chilling susceptibility was evaluated by measuring the root lengths, electrolyte leakage and total dehydrogenase activity.

Root length was determined in Phytotoxkit Microbiotest after seedlings were returned from chilling condition to $25^{\circ} \mathrm{C}$ for $72 \mathrm{~h}$ in a $16 / 8$ photoperiod (Persoone and Vangheluwe 2000). The length was measured with a clear, transparent plastic ruler to the nearest $\mathrm{mm}$.

Twenty-five cucumber roots in four tubes were soaked in $3 \mathrm{ml}$ of distilled water and incubated at $20^{\circ} \mathrm{C}$ for $4 \mathrm{~h}$. The total conductivity of the solution was expressed as the percentages of the total leakage from seeds boiled for $10 \mathrm{~min}$ in water. Results correspond to the means of four measurements. A microcomputer conductivity meter CC-551 (ELMETRON, Poland) was used to measure the electrical conductivities of the leaches of the roots.

For the determination of total dehydrogenase activity, $0.2 \mathrm{~g}$ of root tips were placed in Eppendorf tubes, ground and incubated in $1 \mathrm{~cm}^{3}$ of $0.1 \mathrm{M}$ sodium phosphate buffer, $\mathrm{pH} 7.2$ containing $0.7 \%$ $(\mathrm{w} / \mathrm{v})$ of 2,3,5-triphenyl tetrazolium chloride at $25^{\circ} \mathrm{C}$ for $24 \mathrm{~h}$. After that time, the samples were centrifuged ( $5 \mathrm{~min} ; 5,000 \times \mathrm{g}$ ) and the pellet was extracted six times with $1 \mathrm{ml}$ of acetone. The solution absorbance was measured at $488 \mathrm{~nm}$. A standard curve was prepared from the known concentration of formazan. Each determination of root viability was made four times.

Each experiments was done twice. The experiment was designed in four replications, each comprising ten seedlings. The differences between the means were estimated by the Duncan multiple range test at a significance level of $p=0.05$.

\section{RESULTS AND DISCUSSION}

Earlier research conducted on sunflower has shown that seed conditioning in water significantly increased seedling tolerance to chilling conditions (Górnik at al. 2014). Furthermore, seedlings obtained from conditioned seeds and subsequently subjected to short-term shock temperatures revealed an additionally increased tolerance to the negative effects of chilling. Preliminary experiments carried out on cucumber have shown that seedling sensitivity of cucumber depended on the length of the roots during which they were subjected to low temperatures (data not shown). Subsequent growth of roots after chilling was often used as one of the best indicators for the evaluation of the severity of chilling injuries (Jennings and Saltveit 1994, Mangrich and Saltveit 2000, Kang and Saltveit 2001, Saltveit 2001, Górnik at al. 2014). Moreover, it has been reported that radicle growth after chilling is an even more sensitive test for seedling tolerance to low temperature than the frequently used measurement of ion leakage 
(Jennings and Saltveit 1994). It was also regarded from preliminary experiments that the chilling sensitive stage of cucumber plant development is when seedlings have $3 \mathrm{~mm}$ of roots.

Present studies have shown that seedling resistance to chilling conditions $\left(0^{\circ} \mathrm{C}\right.$ to for 3 days $)$ depended on the plant growth regulators used during seed imbibition and their concentration as well as the temperature initiating thermal shock and its duration. The highest tolerance to chilling, expressed by subsequent growth of the roots, was observed after seed conditioning in salicylic acid solutions in a concentration of $10^{-4} \mathrm{M}$ for $16 \mathrm{~h}$ at $25^{\circ} \mathrm{C}$, then exposure for $4 \mathrm{~h}$ to a shock temperature of $5^{\circ} \mathrm{C}$ and return to $25^{\circ} \mathrm{C}$ (Tab. 1). Due to such treatment the seedling root length was over 12 times longer than the control ones (imbibed in distilled water and not subjected to short-term temperature impact). Less effective on seedling chilling tolerance was $5^{\circ} \mathrm{C}$ (shock temperature) exposure for $2 \mathrm{~h}$. After this treatment the length of seedling roots was over 10 times longer than the control. Similarly, shock temperature of 35 or $40^{\circ} \mathrm{C}$ also positively affected the obtained seedlings against negative effects of chilling conditions. However, these shock temperatures were not as

Table 1. Root length (mm) of 'Monika' cucumber seedlings after seed conditioning in solutions of salicylic or jasmonic acid or brassinolide for $16 \mathrm{~h}$ at $25^{\circ} \mathrm{C}$, exposure to temperature shock for 1,2 or $4 \mathrm{~h}$ at $0,2.5,5,35,40$ or $45^{\circ} \mathrm{C}$ and returning to $25^{\circ} \mathrm{C}$. Control seeds were conditioned in distilled water at $25^{\circ} \mathrm{C}$ with no temperature shock. Seedlings with $3 \mathrm{~mm}$ roots were chilled at $0^{\circ} \mathrm{C}$ for 3 days. Root length was determined after seedlings were returned from chilling conditions to $25^{\circ} \mathrm{C}$ for $72 \mathrm{~h}$

\begin{tabular}{|c|c|c|c|c|c|c|c|c|c|}
\hline \multirow{3}{*}{$\begin{array}{l}\text { Short-term } \\
\text { shock } \\
\text { temperature } \\
\left({ }^{\circ} \mathrm{C}\right)\end{array}$} & \multicolumn{9}{|c|}{ Root length (mm) } \\
\hline & \multicolumn{9}{|c|}{ Duration of shock temperature (h) } \\
\hline & 1 & 2 & 4 & 1 & 2 & 4 & 1 & 2 & 4 \\
\hline & \multicolumn{9}{|c|}{ Concentration of salicylic acid } \\
\hline & \multicolumn{3}{|c|}{$10^{-2} \mathrm{M}$} & \multicolumn{3}{|c|}{$10^{-3} \mathrm{M}$} & \multicolumn{3}{|c|}{$10^{-4} \mathrm{M}$} \\
\hline Control & $3.1 \mathrm{a}$ & $3.1 \mathrm{a}$ & $3.1 \mathrm{a}$ & $3.1 \mathrm{a}$ & $3.1 \mathrm{a}$ & $3.1 \mathrm{a}$ & $3.1 \mathrm{a}$ & $3.1 \mathrm{a}$ & $3.1 \mathrm{a}$ \\
\hline 0 & $3.2 \mathrm{a}$ & $3.1 \mathrm{a}$ & $3.7 \mathrm{a}$ & $3.8 \mathrm{a}$ & $3.8 \mathrm{a}$ & $4.1 \mathrm{~b}$ & $3.7 \mathrm{a}$ & $4.3 \mathrm{~b}$ & $4.6 \mathrm{~b}$ \\
\hline 2.5 & $3.0 \mathrm{a}$ & $3.0 \mathrm{a}$ & $3.0 \mathrm{a}$ & $3.6 \mathrm{a}$ & $5.3 \mathrm{c}$ & $6.9 \mathrm{~d}$ & $5.4 \mathrm{~b}$ & $8.0 \mathrm{~d}$ & $8.1 \mathrm{~d}$ \\
\hline 5 & $3.0 \mathrm{a}$ & $3.0 \mathrm{a}$ & $3.0 \mathrm{a}$ & $17.7 \mathrm{c}$ & $21.2 \mathrm{e}$ & $23.9 \mathrm{e}$ & $28.0 \mathrm{e}$ & $31.6 \mathrm{e}$ & $38.9 \mathrm{f}$ \\
\hline 35 & $3.0 \mathrm{a}$ & $3.0 \mathrm{a}$ & $3.0 \mathrm{a}$ & $3.0 \mathrm{a}$ & $4.3 \mathrm{~b}$ & $4.5 \mathrm{~b}$ & $7.3 \mathrm{c}$ & $7.6 \mathrm{~d}$ & $10.5 \mathrm{e}$ \\
\hline 40 & $3.0 \mathrm{a}$ & $3.0 \mathrm{a}$ & $3.0 \mathrm{a}$ & $5.7 \mathrm{~b}$ & $6.3 \mathrm{~d}$ & $6.8 \mathrm{c}$ & $7.3 \mathrm{c}$ & $8.5 \mathrm{~d}$ & $10.6 \mathrm{e}$ \\
\hline \multirow[t]{3}{*}{45} & $3.0 \mathrm{a}$ & $3.0 \mathrm{a}$ & $3.0 \mathrm{a}$ & $5.1 \mathrm{~b}$ & $5.2 \mathrm{~b}$ & $5.9 \mathrm{c}$ & $5.0 \mathrm{~b}$ & $5.7 \mathrm{c}$ & $6.2 \mathrm{c}$ \\
\hline & \multicolumn{9}{|c|}{ Concentration of jasmonic acid } \\
\hline & \multicolumn{3}{|c|}{$10^{-2} \mathrm{M}$} & \multicolumn{3}{|c|}{$10^{-3} \mathrm{M}$} & \multicolumn{3}{|c|}{$10^{-4} \mathrm{M}$} \\
\hline Control & $3.1 \mathrm{a}$ & $3.1 \mathrm{a}$ & $3.1 \mathrm{a}$ & $3.1 \mathrm{a}$ & $3.1 \mathrm{a}$ & $3.1 \mathrm{a}$ & $3.1 \mathrm{a}$ & $3.1 \mathrm{a}$ & $3.1 \mathrm{a}$ \\
\hline 0 & $7.0 \mathrm{~b}$ & $7.5 \mathrm{~b}$ & $8.6 \mathrm{c}$ & $10.8 \mathrm{~d}$ & $11.2 \mathrm{~d}$ & $11.3 \mathrm{c}$ & $13.3 \mathrm{~d}$ & $14.3 \mathrm{~d}$ & $21.8 \mathrm{e}$ \\
\hline 2.5 & $8.8 \mathrm{c}$ & $9.2 \mathrm{c}$ & $9.6 \mathrm{~d}$ & $7.1 \mathrm{c}$ & $10.8 \mathrm{~d}$ & $11.0 \mathrm{~b}$ & $14.0 \mathrm{~d}$ & $14.0 \mathrm{~d}$ & $14.8 \mathrm{c}$ \\
\hline 5 & $7.6 \mathrm{c}$ & $11.1 \mathrm{~d}$ & $11.2 \mathrm{e}$ & $6.6 \mathrm{bc}$ & $8.7 \mathrm{c}$ & $10.5 \mathrm{~b}$ & $12.4 \mathrm{c}$ & $13.1 \mathrm{c}$ & $14.6 \mathrm{c}$ \\
\hline 35 & $6.6 \mathrm{~b}$ & $7.1 \mathrm{~b}$ & $7.2 \mathrm{~b}$ & $5.8 \mathrm{~b}$ & $7.7 \mathrm{~b}$ & $10.1 \mathrm{~b}$ & $12.0 \mathrm{c}$ & $12.6 \mathrm{c}$ & $13.3 \mathrm{~b}$ \\
\hline 40 & $11.9 \mathrm{~d}$ & $12.9 \mathrm{e}$ & $13.2 \mathrm{f}$ & $10.1 \mathrm{~d}$ & $12.6 \mathrm{e}$ & $14.0 \mathrm{~d}$ & $13.3 \mathrm{~d}$ & $15.8 \mathrm{e}$ & $18.4 \mathrm{~d}$ \\
\hline \multirow[t]{3}{*}{45} & $3.0 \mathrm{a}$ & $3.0 \mathrm{a}$ & $3.0 \mathrm{a}$ & $3.1 \mathrm{a}$ & $3.1 \mathrm{a}$ & $3.5 \mathrm{a}$ & $8.7 \mathrm{~b}$ & $8.8 \mathrm{~b}$ & $10.5 \mathrm{~b}$ \\
\hline & \multicolumn{9}{|c|}{ Concentration of brassinolide } \\
\hline & \multicolumn{3}{|c|}{$10^{-6} \mathrm{M}$} & \multicolumn{3}{|c|}{$10^{-8} \mathrm{M}$} & \multicolumn{3}{|c|}{$10^{-10} \mathrm{M}$} \\
\hline Control & $3.1 \mathrm{a}$ & $3.1 \mathrm{a}$ & $3.1 \mathrm{a}$ & $3.1 \mathrm{a}$ & $3.1 \mathrm{a}$ & $3.1 \mathrm{a}$ & $3.1 \mathrm{a}$ & $3.1 \mathrm{a}$ & $3.1 \mathrm{a}$ \\
\hline 0 & $3.0 \mathrm{a}$ & $3.0 \mathrm{a}$ & $3.8 \mathrm{a}$ & $5.1 \mathrm{~b}$ & $5.8 \mathrm{~b}$ & $6.0 \mathrm{c}$ & $9.4 \mathrm{~d}$ & $10.7 \mathrm{~d}$ & $11.9 \mathrm{~d}$ \\
\hline 2.5 & $3.1 \mathrm{a}$ & $3.2 \mathrm{a}$ & $3.4 \mathrm{a}$ & $6.4 \mathrm{c}$ & $7.4 \mathrm{c}$ & $7.4 \mathrm{~d}$ & $10.7 \mathrm{e}$ & $12.9 \mathrm{e}$ & $20.0 \mathrm{f}$ \\
\hline 5 & $3.1 \mathrm{a}$ & $3.1 \mathrm{a}$ & $3.2 \mathrm{a}$ & $5.8 \mathrm{~b}$ & $6.5 \mathrm{~b}$ & $6.7 \mathrm{c}$ & $13.0 \mathrm{f}$ & $13.5 \mathrm{e}$ & $15.4 \mathrm{e}$ \\
\hline 35 & $3.1 \mathrm{a}$ & $3.1 \mathrm{a}$ & $3.4 \mathrm{a}$ & $3.6 \mathrm{a}$ & $3.7 \mathrm{a}$ & $3.8 \mathrm{a}$ & $6.4 \mathrm{~b}$ & $7.8 \mathrm{~b}$ & $8.9 \mathrm{~b}$ \\
\hline 40 & $3.2 \mathrm{a}$ & $3.6 \mathrm{a}$ & $3.8 \mathrm{a}$ & $3.4 \mathrm{a}$ & $3.8 \mathrm{a}$ & $4.2 \mathrm{~b}$ & $8.3 \mathrm{c}$ & $8.6 \mathrm{~b}$ & $8.7 \mathrm{~b}$ \\
\hline 45 & $3.0 \mathrm{a}$ & $3.0 \mathrm{a}$ & $3.0 \mathrm{a}$ & $3.1 \mathrm{a}$ & $3.1 \mathrm{a}$ & $3.5 \mathrm{a}$ & $8.7 \mathrm{c}$ & $8.8 \mathrm{c}$ & $10.5 \mathrm{c}$ \\
\hline
\end{tabular}

Means within the same substance and columns marked with the same letter are not significantly different at $\mathrm{p}=0.05$ according to the Duncan multiple range test 
protective as $5^{\circ} \mathrm{C}$. The positive effect of salicylic acid in alleviating the negative effects of chilling may be caused by the accumulation of abscisic acid (ABA), which might contribute to a preadaptation of plants to stress, since ABA is known to play a key role in the induction of the synthesis of stress protein (Shakirova at al. 2003). Salicylic acid also caused a significant increase in the concentration of proline in wheat seedlings, which is implicated in the mechanism of the action of SA (Shakirova at al. 2003). The present study revealed that low temperature $\left(5^{\circ} \mathrm{C}\right)$ exposure for $4 \mathrm{~h}$ after $16 \mathrm{~h}$ of seed imbibition increased seedling tolerance to chilling (Tab. 1). Apparently, plants develop the ability to withstand lethal temperatures upon exposure to sub-lethal temperatures due to such treatment (referred as induction stress) (Senthil-Kumar et al. 2008).

The present research also clearly demonstrated that cucumber seed conditioning in jasmonic acid solutions in a concentration of $10^{-4} \mathrm{M}$ for $16 \mathrm{~h}$ at $25^{\circ} \mathrm{C}$, then exposure for $1-4 \mathrm{~h}$ to a shock temperature of $0,2.5,5,35,40$ or $45^{\circ} \mathrm{C}$ resulted in a higher resistance to chilling conditions compared to the control (Tab. 1). In the presence of jasmonic acid in a concentration of $10^{-4} \mathrm{M}$, the best effect was observed after exposing conditioned seeds to $0^{\circ} \mathrm{C}$ for $4 \mathrm{~h}$. Despite the adverse impact of chilling

Table 2. Electrolyte leakage of cucumber seedlings obtained from seeds conditioned in solutions of salicylic or jasmonic acid or brassinolide for $16 \mathrm{~h}$ at $25^{\circ} \mathrm{C}$, temperature shocked at $0,2.5,5,35,40$ or $45^{\circ} \mathrm{C}$ for 1,2 or $4 \mathrm{~h}$ and returned to $25^{\circ} \mathrm{C}$. Control seeds were conditioned at $25^{\circ} \mathrm{C}$ in water and not subjected to short-term shock temperature. Seedlings with $3 \mathrm{~mm}$ roots were chilled at $0^{\circ} \mathrm{C}$ for 3 days

\begin{tabular}{|c|c|c|c|c|c|c|c|c|c|}
\hline \multirow{3}{*}{$\begin{array}{l}\text { Short-term } \\
\text { shock } \\
\text { temperature } \\
\left({ }^{\circ} \mathrm{C}\right)\end{array}$} & \multicolumn{9}{|c|}{ Electrolyte leakage $\left(\mu \mathrm{S} \cdot 25\right.$ seedling roots $\left.\cdot 5 \mathrm{ml} \mathrm{H}_{2} \mathrm{O}\right)$} \\
\hline & \multicolumn{9}{|c|}{ Duration of shock temperature $(\mathrm{h})$} \\
\hline & 1 & 2 & 4 & 1 & 2 & 4 & 1 & 2 & 4 \\
\hline & \multicolumn{9}{|c|}{ Concentration of salicylic acid } \\
\hline & \multicolumn{3}{|c|}{$10^{-2} \mathrm{M}$} & \multicolumn{3}{|c|}{$10^{-3} \mathrm{M}$} & \multicolumn{3}{|c|}{$10^{-4} \mathrm{M}$} \\
\hline Control & $57.2 \mathrm{~b}$ & $57.2 \mathrm{~b}$ & $57.2 \mathrm{~b}$ & $57.2 \mathrm{~d}$ & $57.2 \mathrm{~d}$ & $57.2 \mathrm{e}$ & $57.2 \mathrm{~d}$ & $57.2 \mathrm{~d}$ & $57.2 \mathrm{~d}$ \\
\hline 0 & $55.4 \mathrm{a}$ & $57.2 \mathrm{a}$ & $53.1 \mathrm{a}$ & $55.2 \mathrm{c}$ & $57.0 \mathrm{~d}$ & $50.9 \mathrm{c}$ & $57.2 \mathrm{~d}$ & $55.1 \mathrm{~d}$ & $54.7 \mathrm{~d}$ \\
\hline 2.5 & $55.5 \mathrm{a}$ & $56.8 \mathrm{~b}$ & $54.5 \mathrm{a}$ & $55.6 \mathrm{c}$ & $51.3 \mathrm{c}$ & $47.6 \mathrm{~b}$ & $51.1 \mathrm{c}$ & $46.4 \mathrm{~b}$ & $45.3 \mathrm{c}$ \\
\hline 5 & $56.2 \mathrm{a}$ & $56.3 \mathrm{a}$ & $56.8 \mathrm{~b}$ & $46.0 \mathrm{a}$ & $40.2 \mathrm{a}$ & $38.3 \mathrm{a}$ & $35.2 \mathrm{a}$ & $31.3 \mathrm{a}$ & $27.3 \mathrm{a}$ \\
\hline 35 & $57.6 \mathrm{a}$ & $55.5 \mathrm{a}$ & $54.6 \mathrm{a}$ & $59.0 \mathrm{~d}$ & $55.2 \mathrm{~d}$ & $54.1 \mathrm{~d}$ & $47.2 \mathrm{~b}$ & $47.1 \mathrm{~b}$ & $42.5 \mathrm{~b}$ \\
\hline 40 & $56.3 \mathrm{a}$ & $55.5 \mathrm{a}$ & $56.8 \mathrm{~b}$ & $52.6 \mathrm{~b}$ & $48.5 \mathrm{~b}$ & $47.4 \mathrm{~b}$ & $47.1 \mathrm{~b}$ & $45.4 \mathrm{~b}$ & $42.1 \mathrm{~b}$ \\
\hline \multirow[t]{3}{*}{45} & $55.4 \mathrm{a}$ & $55.5 \mathrm{a}$ & $55.3 \mathrm{a}$ & $50.3 \mathrm{~b}$ & $49.6 \mathrm{~b}$ & $48.3 \mathrm{~b}$ & $49.4 \mathrm{~b}$ & $50.2 \mathrm{c}$ & $48.3 \mathrm{c}$ \\
\hline & \multicolumn{9}{|c|}{ Concentration of jasmonic acid } \\
\hline & \multicolumn{3}{|c|}{$10^{-2} \mathrm{M}$} & \multicolumn{3}{|c|}{$10^{-3} \mathrm{M}$} & \multicolumn{3}{|c|}{$10^{-4} \mathrm{M}$} \\
\hline Control & $57.2 \mathrm{c}$ & $57.2 \mathrm{c}$ & $57.2 \mathrm{c}$ & $57.2 \mathrm{c}$ & $57.2 \mathrm{c}$ & $57.2 \mathrm{c}$ & $57.2 \mathrm{c}$ & $57.2 \mathrm{~d}$ & $57.2 \mathrm{c}$ \\
\hline 0 & $47.4 \mathrm{~b}$ & $46.7 \mathrm{~b}$ & $46.1 \mathrm{~b}$ & $43.0 \mathrm{a}$ & $43.2 \mathrm{a}$ & $43.3 \mathrm{~b}$ & $39.9 \mathrm{a}$ & $39.1 \mathrm{a}$ & $38.2 \mathrm{a}$ \\
\hline 2.5 & $45.3 \mathrm{~b}$ & $44.6 \mathrm{a}$ & $44.2 \mathrm{~b}$ & $47.1 \mathrm{~b}$ & $42.8 \mathrm{a}$ & $43.0 \mathrm{~b}$ & $39.1 \mathrm{a}$ & $39.4 \mathrm{a}$ & $39.0 \mathrm{a}$ \\
\hline 5 & $46.5 \mathrm{~b}$ & $43.1 \mathrm{a}$ & $43.0 \mathrm{~b}$ & $48.8 \mathrm{~b}$ & $45.2 \mathrm{a}$ & $42.8 \mathrm{~b}$ & $42.0 \mathrm{~b}$ & $40.2 \mathrm{~b}$ & $39.2 \mathrm{a}$ \\
\hline 35 & $46.8 \mathrm{~b}$ & $47.2 \mathrm{~b}$ & $46.9 \mathrm{~b}$ & $51.6 \mathrm{bc}$ & $46.4 \mathrm{~b}$ & $43.3 \mathrm{~b}$ & $42.8 \mathrm{~b}$ & $40.9 \mathrm{~b}$ & $39.7 \mathrm{a}$ \\
\hline 40 & $42.8 \mathrm{a}$ & $41.1 \mathrm{a}$ & $40.8 \mathrm{a}$ & $43.3 \mathrm{~b}$ & $42.0 \mathrm{a}$ & $39.2 \mathrm{a}$ & $40.8 \mathrm{~b}$ & $40.1 \mathrm{~b}$ & $39.3 \mathrm{a}$ \\
\hline \multirow[t]{3}{*}{45} & $57.5 \mathrm{c}$ & $57.8 \mathrm{c}$ & $57.2 \mathrm{c}$ & $57.4 \mathrm{c}$ & $57.6 \mathrm{c}$ & $56.8 \mathrm{c}$ & $56.2 \mathrm{c}$ & $45.3 \mathrm{c}$ & $42.8 \mathrm{~b}$ \\
\hline & \multicolumn{9}{|c|}{ Concentration of brassinolide } \\
\hline & \multicolumn{3}{|c|}{$10^{-6} \mathrm{M}$} & \multicolumn{3}{|c|}{$10^{-8} \mathrm{M}$} & \multicolumn{3}{|c|}{$10^{-10} \mathrm{M}$} \\
\hline Control & $57.2 \mathrm{a}$ & $57.2 \mathrm{a}$ & $57.2 \mathrm{a}$ & $57.2 \mathrm{~b}$ & $57.2 \mathrm{c}$ & $57.2 \mathrm{~b}$ & $57.2 \mathrm{c}$ & $57.2 \mathrm{c}$ & $57.2 \mathrm{c}$ \\
\hline 0 & $57.8 \mathrm{a}$ & $58.8 \mathrm{a}$ & $59.1 \mathrm{a}$ & $51.6 \mathrm{a}$ & $49.4 \mathrm{~b}$ & $49.0 \mathrm{a}$ & $47.3 \mathrm{~b}$ & $45.2 \mathrm{~b}$ & $40.3 \mathrm{ab}$ \\
\hline 2.5 & $57.0 \mathrm{a}$ & $57.6 \mathrm{a}$ & $58.4 \mathrm{a}$ & $48.5 \mathrm{a}$ & $47.4 \mathrm{~b}$ & $47.5 \mathrm{a}$ & $45.3 \mathrm{~b}$ & $41.0 \mathrm{a}$ & $37.6 \mathrm{a}$ \\
\hline 5 & $57.9 \mathrm{a}$ & $57.8 \mathrm{a}$ & $57.9 \mathrm{a}$ & $49.6 \mathrm{a}$ & $51.4 \mathrm{ab}$ & $51.0 \mathrm{ab}$ & $40.3 \mathrm{a}$ & $39.5 \mathrm{a}$ & $37.6 \mathrm{a}$ \\
\hline 35 & $57.6 \mathrm{a}$ & $57.5 \mathrm{a}$ & $58.2 \mathrm{a}$ & $59.1 \mathrm{~b}$ & $59.0 \mathrm{c}$ & $59.4 \mathrm{~b}$ & $51.5 \mathrm{bc}$ & $48.6 \mathrm{~b}$ & $47.3 \mathrm{~b}$ \\
\hline 40 & $57.6 \mathrm{a}$ & $58.3 \mathrm{a}$ & $59.2 \mathrm{a}$ & $58.5 \mathrm{~b}$ & $59.3 \mathrm{c}$ & $55.0 \mathrm{~b}$ & $48.2 \mathrm{~b}$ & $47.5 \mathrm{~b}$ & $47.4 \mathrm{~b}$ \\
\hline 45 & $56.2 \mathrm{a}$ & $56.2 \mathrm{a}$ & $57.0 \mathrm{a}$ & $57.6 \mathrm{~b}$ & $57.4 \mathrm{c}$ & $58.4 \mathrm{~b}$ & $47.5 \mathrm{~b}$ & $47.6 \mathrm{~b}$ & $45.3 \mathrm{~b}$ \\
\hline
\end{tabular}

Means within the same substance and columns marked with the same letter are not significantly different at $p=0.05$ according to the Duncan multiple range test 
conditions, jasmonic acid and shock temperature exposure stimulated the growth of roots over $700 \%$ compared with the control plants. Similarly, Korkmaz et al. (2005) found that the inclusion of jasmonic acid into the priming solution improved the low temperature performance of muskmelon seeds.

Seed conditioning in brassinolide in a concentration of $10^{-10} \mathrm{M}$ for $16 \mathrm{~h}$ at $25^{\circ} \mathrm{C}$, afterwards subjecting them for $4 \mathrm{~h}$ to shock temperature of $2.5^{\circ} \mathrm{C}$ or $5^{\circ} \mathrm{C}$, also resulted in increased seedling tolerance to chilling (Tab. 1). Due to such treatments the seedling root length was over five times longer than the control. The spraying of rice leaves with brassinolide also increased plant height and the fresh weights of tops and roots under chilling stress (Bajguz and Hayat 2009).

Previous studies revealed that the integrity of cucumber root membranes measured by electrolyte leakage was impaired by chilling temperatures (data not shown). Similarly, the negative effects of chilling on membrane integrity were visible in the present research in the control seeds, which were conditioned in distilled water at $25^{\circ} \mathrm{C}$ and not subjected to short-term shock temperature (Tab. 2). Posmyk et al. (2001) reported that electrolyte leakage to the medium expressing the integrity of membranes is a highly sensitive test

Table 3. Total dehydrogenase activity of cucumber seedlings obtained from seeds conditioned in solutions of salicylic or jasmonic acid or brassinolide for $16 \mathrm{~h}$ at $25^{\circ} \mathrm{C}$, temperature shocked at $0,2.5,5,35,40$ or $45^{\circ} \mathrm{C}$ for 1,2 or $4 \mathrm{~h}$ and returned to $25^{\circ} \mathrm{C}$. Control seeds were conditioned at $25^{\circ} \mathrm{C}$ in water and not subjected to short-term shock temperature. Seedlings with $3 \mathrm{~mm}$ roots were chilled at $0^{\circ} \mathrm{C}$ for 3 days

\begin{tabular}{|c|c|c|c|c|c|c|c|c|c|}
\hline \multirow{3}{*}{$\begin{array}{l}\text { Short-term } \\
\text { shock } \\
\text { temperature } \\
\left({ }^{\circ} \mathrm{C}\right)\end{array}$} & \multicolumn{9}{|c|}{ Total dehydrogenase activity ( $\mathrm{mg}$ formazan $\cdot \mathrm{g}^{-1} \mathrm{FW}$ of seeds) } \\
\hline & \multicolumn{9}{|c|}{ Duration of shock temperature $(\mathrm{h})$} \\
\hline & 1 & 2 & 4 & 1 & 2 & 4 & 1 & 2 & 4 \\
\hline & \multicolumn{9}{|c|}{ Concentration of salicylic acid } \\
\hline & \multicolumn{3}{|c|}{$10^{-2} \mathrm{M}$} & \multicolumn{3}{|c|}{$10^{-3} \mathrm{M}$} & \multicolumn{3}{|c|}{$10^{-4} \mathrm{M}$} \\
\hline Control & $0.56^{1} \mathrm{a}$ & $0.56 \mathrm{a}$ & $0.56 \mathrm{a}$ & $0.56 \mathrm{a}$ & $0.56 \mathrm{a}$ & $0.56 \mathrm{a}$ & $0.56 \mathrm{a}$ & $0.56 \mathrm{a}$ & $0.56 \mathrm{a}$ \\
\hline 0 & $0.58 \mathrm{a}$ & $0.50 \mathrm{a}$ & $0.67 \mathrm{a}$ & $0.64 \mathrm{a}$ & $0.69 \mathrm{a}$ & $0.74 \mathrm{a}$ & $0.67 \mathrm{a}$ & $0.78 \mathrm{a}$ & $0.83 \mathrm{a}$ \\
\hline 2.5 & $0.51 \mathrm{a}$ & $0.55 \mathrm{a}$ & $0.59 \mathrm{a}$ & $0.60 \mathrm{a}$ & $0.96 \mathrm{~b}$ & $1.25 \mathrm{~b}$ & $0.98 \mathrm{a}$ & $1.45 \mathrm{~b}$ & $1.46 \mathrm{~b}$ \\
\hline 5 & $0.53 \mathrm{a}$ & $0.54 \mathrm{a}$ & $0.54 \mathrm{a}$ & $3.16 \mathrm{c}$ & $3.83 \mathrm{~d}$ & $4.32 \mathrm{c}$ & $5.06 \mathrm{c}$ & $5.07 \mathrm{~d}$ & $7.03 \mathrm{~d}$ \\
\hline 35 & $0.56 \mathrm{a}$ & $0.56 \mathrm{a}$ & $0.52 \mathrm{a}$ & $0.52 \mathrm{a}$ & $0.78 \mathrm{~b}$ & $0.81 \mathrm{a}$ & $1.32 \mathrm{~b}$ & $1.35 \mathrm{~b}$ & $1.92 \mathrm{c}$ \\
\hline 40 & $0.59 \mathrm{a}$ & $0.62 \mathrm{a}$ & $0.50 \mathrm{a}$ & $1.04 \mathrm{~b}$ & $1.14 \mathrm{c}$ & $1.23 \mathrm{~b}$ & $1.34 \mathrm{~b}$ & $1.53 \mathrm{c}$ & $1.91 \mathrm{c}$ \\
\hline \multirow[t]{3}{*}{45} & $0.60 \mathrm{a}$ & $0.63 \mathrm{a}$ & $0.57 \mathrm{a}$ & $0.92 \mathrm{~b}$ & $0.94 \mathrm{~b}$ & $1.06 \mathrm{~b}$ & $0.91 \mathrm{a}$ & $1.03 \mathrm{~b}$ & $1.12 \mathrm{~b}$ \\
\hline & \multicolumn{9}{|c|}{ Concentration of jasmonic acid } \\
\hline & \multicolumn{3}{|c|}{$10^{-2} \mathrm{M}$} & \multicolumn{3}{|c|}{$10^{-3} \mathrm{M}$} & \multicolumn{3}{|c|}{$10^{-4} \mathrm{M}$} \\
\hline Control & $0.56 \mathrm{a}$ & $0.56 \mathrm{a}$ & $0.56 \mathrm{a}$ & $0.56 \mathrm{a}$ & $0.56 \mathrm{a}$ & $0.56 \mathrm{a}$ & $0.56 \mathrm{a}$ & $0.56 \mathrm{a}$ & $0.56 \mathrm{a}$ \\
\hline 0 & $1.26 \mathrm{~b}$ & $1.35 \mathrm{~b}$ & $1.55 \mathrm{c}$ & $1.95 \mathrm{c}$ & $2.03 \mathrm{~d}$ & $2.04 \mathrm{c}$ & $2.40 \mathrm{~d}$ & $2.62 \mathrm{~d}$ & $3.96 \mathrm{e}$ \\
\hline 2.5 & $1.59 \mathrm{c}$ & $1.66 \mathrm{c}$ & $1.74 \mathrm{~d}$ & $1.31 \mathrm{~b}$ & $1.97 \mathrm{c}$ & $1.92 \mathrm{~b}$ & $2.53 \mathrm{~d}$ & $2.48 \mathrm{~d}$ & $2.62 \mathrm{c}$ \\
\hline 5 & $1.37 \mathrm{~b}$ & $2.00 \mathrm{~d}$ & $2.02 \mathrm{e}$ & $1.21 \mathrm{~b}$ & $1.58 \mathrm{c}$ & $1.89 \mathrm{~b}$ & $2.20 \mathrm{c}$ & $2.35 \mathrm{c}$ & $2.63 \mathrm{c}$ \\
\hline 35 & $1.19 \mathrm{~b}$ & $1.37 \mathrm{~b}$ & $1.30 \mathrm{~b}$ & $1.05 \mathrm{~b}$ & $1.42 \mathrm{~b}$ & $1.82 \mathrm{~b}$ & $2.16 \mathrm{c}$ & $2.25 \mathrm{c}$ & $2.40 \mathrm{~b}$ \\
\hline 40 & $2.15 \mathrm{~d}$ & $2.35 \mathrm{e}$ & $2.38 \mathrm{f}$ & $1.83 \mathrm{c}$ & $2.32 \mathrm{~d}$ & $2.53 \mathrm{~d}$ & $2.40 \mathrm{~d}$ & $2.85 \mathrm{e}$ & $3.32 \mathrm{~d}$ \\
\hline \multirow[t]{3}{*}{45} & $0.54 \mathrm{a}$ & $0.54 \mathrm{a}$ & $0.53 \mathrm{a}$ & $0.50 \mathrm{a}$ & $0.49 \mathrm{a}$ & $0.65 \mathrm{a}$ & $1.53 \mathrm{~b}$ & $1.59 \mathrm{~b}$ & $1.92 \mathrm{~b}$ \\
\hline & \multicolumn{9}{|c|}{ Concentration of brassinolide } \\
\hline & \multicolumn{3}{|c|}{$10^{-6} \mathrm{M}$} & \multicolumn{3}{|c|}{$10^{-8} \mathrm{M}$} & \multicolumn{3}{|c|}{$10^{-10} \mathrm{M}$} \\
\hline Control & $0.56 \mathrm{a}$ & $0.56 \mathrm{a}$ & $0.56 \mathrm{a}$ & $0.56 \mathrm{a}$ & $0.56 \mathrm{a}$ & $0.56 \mathrm{a}$ & $0.56 \mathrm{a}$ & $0.56 \mathrm{a}$ & $0.56 \mathrm{a}$ \\
\hline 0 & $0.53 \mathrm{a}$ & $0.53 \mathrm{a}$ & $0.69 \mathrm{a}$ & $0.92 \mathrm{a}$ & $1.02 \mathrm{~b}$ & $1.14 \mathrm{~b}$ & $0.67 \mathrm{a}$ & $0.79 \mathrm{a}$ & $2.15 \mathrm{~d}$ \\
\hline 2.5 & $0.50 \mathrm{a}$ & $0.51 \mathrm{a}$ & $0.62 \mathrm{a}$ & $1.46 \mathrm{c}$ & $1.31 \mathrm{c}$ & $1.39 \mathrm{c}$ & $0.99 \mathrm{a}$ & $1.48 \mathrm{~b}$ & $3.61 \mathrm{f}$ \\
\hline 5 & $0.59 \mathrm{a}$ & $0.60 \mathrm{a}$ & $0.57 \mathrm{a}$ & $1.05 \mathrm{~b}$ & $1.17 \mathrm{~b}$ & $1.21 \mathrm{~b}$ & $4.79 \mathrm{c}$ & $5.48 \mathrm{~d}$ & $2.68 \mathrm{e}$ \\
\hline 35 & $0.60 \mathrm{a}$ & $0.55 \mathrm{a}$ & $0.65 \mathrm{a}$ & $0.65 \mathrm{a}$ & $0.68 \mathrm{a}$ & $0.68 \mathrm{a}$ & $1.36 \mathrm{~b}$ & $1.28 \mathrm{~b}$ & $1.62 \mathrm{~b}$ \\
\hline 40 & $0.56 \mathrm{a}$ & $0.65 \mathrm{a}$ & $0.66 \mathrm{a}$ & $0.61 \mathrm{a}$ & $0.69 \mathrm{a}$ & $0.76 \mathrm{a}$ & $1.32 \mathrm{~b}$ & $1.55 \mathrm{c}$ & $1.57 \mathrm{~b}$ \\
\hline 45 & $0.52 \mathrm{a}$ & $0.48 \mathrm{a}$ & $0.52 \mathrm{a}$ & $0.57 \mathrm{a}$ & $0.56 \mathrm{a}$ & $0.62 \mathrm{a}$ & $0.95 \mathrm{a}$ & $1.01 \mathrm{~b}$ & $1.98 \mathrm{c}$ \\
\hline
\end{tabular}

Means within the same substance and columns marked with the same letter are not significantly different at $p=0.05$ according to the Duncan multiple range test 
of seedling damage caused by chilling. Saltveit (1989) also stated that the rate of ion leakage was highly correlated with the degree of chilling injury. Bramlage et al. (1978) demonstrated that the leakage of solutes from chilled imbibing seeds suggests that membrane re-organization is impaired at low temperatures. The present results indicate that electrolyte leakage to the medium was positively affected by seed conditioning, short-term shock temperature as well as the examined plant growth regulators (Tab. 2). The lower value of electrolyte leakage measurements indicates the better integrity of the cell membranes of the roots. The presented data indicate that all of the applied plant growth regulators positively affected membrane integrity, protecting it from the damaging effect of chilling. The best effect was visible after seed conditioning in salicylic acid solutions in a concentration of $10^{-4}$ $\mathrm{M}$ for $16 \mathrm{~h}$ at $25^{\circ} \mathrm{C}$, then exposure for $4 \mathrm{~h}$ to a shock temperature of $5^{\circ} \mathrm{C}$.

The obtained results shown that the total dehydrogenase activity of chilled roots, similarly to membrane integrity, was positively influenced by seed conditioning, short-term shock temperature as well as the examined plant growth regulators (Tab. 3). The obtained results reveal that all of the applied plant growth regulators during seed conditioning positively influenced the total dehydrogenase activity. The highest activity of total dehydrogenase of chilled roots was observed after seed conditioning in salicylic acid solutions in a concentration of $10^{-4} \mathrm{M}$ for $16 \mathrm{~h}$ at $25^{\circ} \mathrm{C}$, then exposure for $4 \mathrm{~h}$ to a shock temperature of $5^{\circ} \mathrm{C}$. Higher total dehydrogenase activity of chilled roots suggests that seed conditioning, short-term shock temperature as well as salicylic acid stimulated respiratory enzyme activity in cucumber seedlings. Presumably, an increase of the respiration rate due to such treatments enhanced cucumber chilling tolerance. The activity of dehydrogenases (respiratory enzymes) is considered an index of tissue respiration and metabolism (Kasai et al. 1998, Białecka and Kępczyński 2010, Farooq et al. 2010). In the Kasai et al. (1998) study, an increase in the respiration rate was suggested as an adaptive strategy for stress in Triticum aestivum seeds germinating under salinity.

The present studies have shown that combining the positive impacts of seed conditioning, salicylic or jasmonic acid or brassinolide and short-term shock temperature led to the alleviation to a large degree of the negative effects of chilling on cucumber seedlings. However, this was dependent on the plant growth regulator used during seed imbibition, its concentration as well as the temperature initiating thermal shock and its duration. Further research is needed to explain the mechanism of the positive effects of salicylic acid, jasmonic acid or brassinolide application in reducing the injurious effects of chilling on cucumber seedlings.

\section{CONCLUSIONS}

1. Cucumber seedling resistance to chilling conditions $\left(0^{\circ} \mathrm{C}\right.$ to for 3 days) depends on plant growth regulator used during seed imbibition and its concentration as well as the temperature initiating thermal shock and its duration.

2. The highest cucumber seedling tolerance to chilling, expressed by the subsequent growth of the roots, was observed after seed conditioning in salicylic acid solutions in a concentration of $10^{-4} \mathrm{M}$ for $16 \mathrm{~h}$ at $25^{\circ} \mathrm{C}$, then exposure for $4 \mathrm{~h}$ to a shock temperature of $5^{\circ} \mathrm{C}$.

3. An increased chilling tolerance of cucumber seedlings may result from enhanced membrane integrity and total dehydrogenase activity due to seed conditioning in the plant growth regulator used and short-term shock temperature.

\section{REFERENCES}

Bajguz A., Hayat S., 2009. Effects of brassinosteroids on the plant responses to environmental stresses. Plant Physiol. Biochem. 47: 1-8.

BiAŁeckA B., KęPCZYŃSKi J., 2010. Germination, $\alpha$-, $\beta$-amylase and total dehydrogenase activities of Amaranthus caudatus seeds under water stress in the presence of ethephon or gibberellin A3. Acta Biol. Cracov. Ser. Bot. 52: 7-12.

Bramlage W.J., Leopold A.C., Parrish D.T., 1978. Chilling stress to soybeans during imbibition. Plant Physiol. 61: 525-529.

Collins G.G., Nie X.L., Saltveit M.L., 1993. Heat shock increases chilling tolerance of mung bean hypocotyl tissue. Physiol. Plant. 89(1): 117-124.

Faroog M., Wahid A., Ahmad N., Asad S.A., 2010. Comparative efficacy of surface drying and redrying seed priming in rice: changes in emergence, seedling growth and associated metabolic events. Paddy Water. Environ. 8: 15-22.

Górnik K., BAdowiec A., Weidner W., 2014. The effect of seed conditioning, short-term heat shock and salicylic, jasmonic acid or brasinolide on sunflower (Helianthus annuus L.) chilling resistance and polysome formation. Acta Physiol. Plant. DOI 10.1007/s11738-014-1626-5. 
Jennings P., SALEveit M.E., 1994. Temperature effects on imbibition and germination of cucumber (Cucumis sativus) seeds. J. Am. Soc. Hortic. Sci. 119: 464-467.

KaCPERSKA A., 2002. Reakcje roślin na abiotyczne czynniki stresowe. In: Fizjologia roślin. J. Kopcewicz and S. Lewak (eds), Wydawnictwo Naukowe PWN, Warszawa: 637-648.

Kang H., Saltveit M.E., 2001. Activity of enzymatic antioxidant defense systems in chilled and heat shocked cucumber seedling radicles. Physiol. Plant. 113: 548-556.

Kang H.M, SAltveit M.E., 2002. Effect of chilling on antioxidant enzymes and DPPH-radical scavenging activity of high- and low-vigour cucumber seedling radicles. Plant Cell Environ. 25: 1233-1238.

Kasai K., Mori N., NaKamura C., 1998. Changes in the respiratory pathways during germination and early seedling growth of common wheat under normal and $\mathrm{NaCl}$ stressed conditions. Cereal Res. Commun. 26: 217-224.

Korkmaz A., Ozbay N, Tiryaki I., Nas M.N., 2005. Combining priming and plant growth regulators improves muskmelon germination and emergence at low temperatures. Eur. J. Hortic. Sci. 70: 29-34.

Mangrich M.E., SAltveit M.E., 2000. Effect of chilling, heat shock, and vigour on the growth of cucumber (Cucumis sativus) radicles. Physiol. Plant. 109(2): 137-142.

Persoone G., Vangheluwe M.L., 2000. Toxicity determination of the sediments of the river Seine in France by application of a battery of microbiotests. In: New Microbiotests for Routine Toxicity Screening and Biomonitoring. G. Persoone, C. Janssen and
W. De Coen (eds), Kluwer Academic, New York: 427- 439.

Posmyk M., Corbineau F., Vinel D, Bailly C., Come D., 2001. Osmoconditioning reduces physiological and biochemical damage induced by chilling in soybean seeds. Physiol. Plant. 111: 473-482.

SAltveit M.E., 1989. A kinetic examination of iron leakage from chilled tomato pericarp discs. Acta Hortic. 258: 617-622.

SAltveit M.E., 1991. Prior temperature exposure affects subsequent chilling sensitivity. Physiol. Plant. 113: 548-556.

Saltveit M.E., 2001. Chilling injury is reduced in cucumber and rice seedlings and in tomato pericarp discs by heat-shocks applied after chilling. Postharvest Biol. Tec. 21: 169-177.

Senthil-Kumar M., Srikanthbabu V., Mohan Raju B., Ganeshimuar, Shivaprakash N., Udayakumar M., 2003. Screening of inbred lines to develop a thermotolerant sunflower hybrid using the temperature induction response (TIR) technique: a novel approach by exploiting residual variability. J. Exp. Bot. 54, 392: 2569-2578.

Shakirowa F.M, Sakhabutdinova A.R, Bezrukova M.V, Fatkhutdinova RA, Fatkhutdinova D.R., 2003. Changes in the hormonal status of wheat seedlings induced by salicylic acid and salinity. Plant Sci. 164: 317-322.

Stark Z., Choluj D., Nemyska B., 1993. Fizjologiczne reakcje roślin na niekorzystne czynniki środowiska. Wydawnictwo SGGW, Warszawa.

Received August 29, 2014; accepted November 19, 2014 